\title{
Specificity, transfer, and the development of expertise
}

\author{
David T. Brookes \\ Department of Physics, Florida International University, 11200 SW 8th Street CP 204, Miami, Florida 33199, USA
}

Brian H. Ross

Department of Psychology, University of Illinois at Urbana-Champaign, 603 East Daniel Street, Champaign, Illinois 61820, USA

José P. Mestre

Department of Physics and Educational Psychology, Loomis Laboratory of Physics, 1110 West Green Street, Urbana, Illinois 61801-3080, USA

(Received 7 August 2010; published 7 April 2011)

\begin{abstract}
In this paper we present the results of two experiments designed to understand how physics students' learning of the concept of refraction is influenced by the cognitive phenomenon of "specificity." In both experiments participants learned why light bends as it travels from one optical medium to another with an analogy made to a car driving from paved road into mud and vice versa. They then learned how to qualitatively draw the direction of refracted light rays with an example of a glass prism. One group learned with a rectangular prism example while a second group learned with a triangular prism example. In a transfer test, the participants revealed how, even when they seemed able to implement the refraction concept, their responses were biased by the example they had seen. Participants frequently violated the refraction principle they had just learned (reversing the bend direction) in order to make sure their response matched the surface features of their learning example. This tended to happen when their test question looked superficially similar to their learning example. We discuss the implications of these results for physics instruction.
\end{abstract}

DOI: 10.1103/PhysRevSTPER.7.010105

PACS numbers: 01.40.Fk, 01.40.Ha

\section{INTRODUCTION}

How and what do students learn from the examples we show them in a physics class? In physics we often explain abstract physics principles to students. We then apply the principle to one or more concrete examples. We hope that students will develop a deeper understanding of the physics principle and how it is connected to real-life situations. We hope that students will be able to generalize their knowledge to many situations, displaying evidence of transfer.

In cognitive science, Ross showed that if the content of the new situation is similar to the training example used to illustrate the principle, then the features of this new content become a strong cue for students to use the same method or procedure as they learned in the training example. Even when students understand a particular principle, students' understanding of the principle becomes bound up in the particulars of the example that is used to illustrate the principle [1-5]. This phenomenon is known as the "specificity effect."

Published by American Physical Society under the terms of the Creative Commons Attribution 3.0 License. Further distribution of this work must maintain attribution to the author(s) and the published article's title, journal citation, and DOI.
The question we want to ask is, How do students use prior examples that they have seen in their physics course to solve new problems? More specifically, can we see evidence for the specificity effect in learning physics? Answering this question is ultimately an issue of transfer. It is critical for understanding the dynamics of the transfer process. One possibility is that in order to successfully solve a problem, a student needs to recognize what sort of a problem it is (e.g., "this is an energy conservation problem") and transfer their principled understanding [6]. Another possibility is the analogy or similarity view. In this view, students cue ideas based on the similarity between the target problem and features of prior examples in which the principle has been applied. In reality, it is probably not an either/or situation [7]. Research in problem solving and transfer has also shown that students transfer surface features of examples they have seen before into new situations [8]. But, Podolefsky and Finkelstein, using their analogical scaffolding model, have shown that students blend representations together to make new ones, and may transfer deeper structure into new situations as part of a blended representation [9].

We believe that the specificity effect is common in physics learning and could have unexpected consequences for what students learn and how they apply physics principles in new situations. We will present two experiments 
that we conducted to test for the presence of this effect. Based on the specificity hypothesis, we predicted that even when students are able to implement the principle, the surface features of a particular testing situation (if similar enough to their learning example) could override their principle knowledge. In such cases, students' solutions would be heavily influenced by the particular learning example, though they can apply the principle correctly to other problems.

As we will show in this paper, the outcomes of the experiments that we conducted not only supported the hypothesis of specificity in student reasoning, but provided us with interesting insights into possible factors that increase or decrease the incidence of this effect.

\section{METHOD}

\section{A. Participants}

In experiment 1 we recruited 31 undergraduate physics students from a large Midwestern university to learn about refraction. All students were in their first semester of an introductory algebra-based physics course for life-science majors and premeds. Participants were randomly assigned to one of two learning conditions that each used a different illustrative example, a triangle prism (T-trained, $n=15$ ) or a rectangle prism (R-trained, $n=16$ ). In experiment 2 we recruited 48 undergraduate physics students to learn about refraction. The students were in their first semester of a calculus-based physics course for engineers and physics majors. As before, students were randomly assigned to either the triangle learning condition $(n=25)$ or the rectangle learning condition $(n=23)$.

\section{B. Materials and procedures}

In order to help participants learn about refraction we engaged them in a 20-min instructional sequence.

(1) Participants saw a demonstration of the actual physical apparatus of the prism and refraction of a laser beam through the prism. In the rectangle condition, participants saw a laser beam being refracted through a rectangular prism. In the triangle condition, participants saw a laser beam being refracted through an equilateral triangular prism.

(2) Participants learned that light was a wave and the instructor provided an analogy to water waves traveling towards a beach so that they could visualize in the case of electromagnetic plane waves - the orientation between the wave fronts and the direction of travel. They were able to visualize the light from the laser beam in a similar way using this analogy.

(3) The instructor then introduced the idea that light travels at different speeds in different optical media. Students learned that the refractive index of a particular medium is expressed as the ratio of the speed of light in the vacuum to the speed of light in that medium.
(4) The instructor offered participants an analogy involving a car slowing down as it transitioned from paved road to mud and speeding up as it transitioned from mud to paved road. Whichever wheel entered or exited the mud first would slow down or speed up first and cause the car to twist in the appropriate direction.

(5) Finally, participants were asked to apply the car analogy to the case of refraction, applying it to either the rectangle prism (R-trained group) or the triangle prism (T-trained group). After a short discussion, the instructor challenged students to fix their work if they made mistakes before finally modeling for them [10] how to apply refraction using the car analogy. Figure 1 shows the learning examples used in experiment 1 . Figure 2 shows the modified examples used in experiment 2 . In all cases, when the instructor modeled drawing the rays in the example, wave fronts were inserted in the diagram to help participants make connections to the analogy. Normal lines were also drawn at each prism interface.

In experiment 1 , this learning phase was followed by a four-question test given to all participants. In each question, students were asked to complete the light ray and extend their exiting ray until it intersected with the screen. The test questions are shown in Table I. Question 4 was included to assess whether participants could apply the principle of refraction at a single interface. No screen was provided in question 4 . In question 1 students were

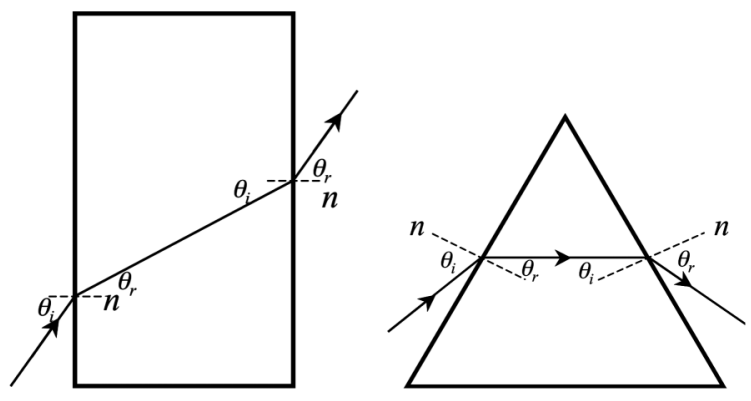

FIG. 1. Rectangle and triangle training examples for experiment 1 .

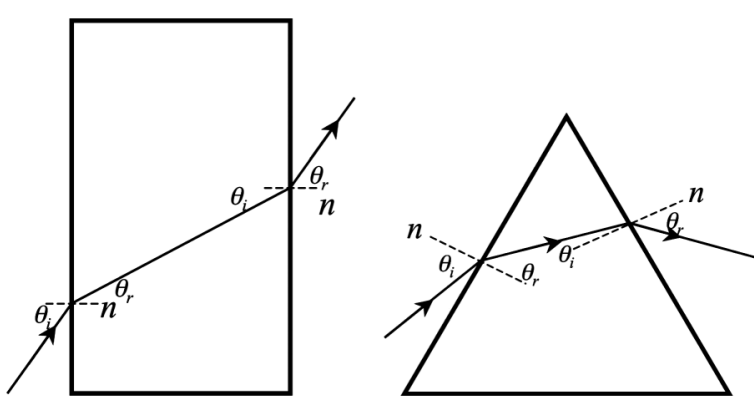

FIG. 2. Rectangle and triangle training examples for experiment 2. Note that the triangle example was modified from experiment 1 for reasons that are explained later in the text. 
TABLE I. The four assessment questions for experiment 1. The order of presentation of questions 2 and 3 was counterbalanced. Participants were not allowed to return to a previous question once they had completed it and gone on to the next question.

Question 2
Rectangle prism

told that the $D$ prism was a half-circle and $C$ marked the center of the circle.

In experiment 2 , the instructional phase was followed by a five-question test. The instructions were the same, but the test questions were modified from experiment 1 . The test questions are shown in Table II. Here, question 5 was used as our criterion as to whether the student could apply the principle of refraction. In contrast with question 4 of experiment 1 , no normal line was provided to help students.

The presence of the screens requires explanation: The screens were placed in the questions because we were initially unsure how we were going to measure the biases in how participants drew the light rays. In the end, the screens turned out to be unnecessary as we simply used a protractor to measure the angle between the incident or refracted rays and the normal line at each interface.

\section{RESULTS AND ANALYSIS}

\section{A. What did students think about the instruction?}

In a written posttest survey, we found that participants were enthusiastic about the pavement-mud analogy and thought that it really helped them understand the principle of refraction. One of the questions asked subjects, "Do you have any comments about the way you were taught today or the problems you were asked to solve?" Some typical participant responses were as follows.

"Yes, the analogy helped a lot. It made it much easier to visualize the solution."

TABLE II. The five assessment questions for experiment 2. The order of presentation of questions 1 and 2, and questions 3 and 4 , was counterbalanced. Participants were not allowed to return to a previous question once they had completed it and gone on to the next question.

\begin{tabular}{lll}
\hline \hline Question 1 & $\begin{array}{c}\text { Question 2 } \\
\text { Rectangle prism }\end{array}$ \\
\hline Triangle prism & Question 3
\end{tabular}


TABLE III. Summary of the angles and measures for participants in experiment 1.

\begin{tabular}{lccccccc}
\hline \hline & \multicolumn{2}{c}{ Question 1, $D$ prism } & \multicolumn{2}{c}{ Question 2, Rectangle prism } & \multicolumn{2}{c}{ Question 3, Triangle prism } & Question 4, Single interface \\
Interface & Air-Glass & Glass-Air & Air-Glass & Glass-Air & Air-Glass & Glass-Air & Glass-Air \\
Measure & $\theta_{\mathrm{r}}$ & $n_{\mathrm{g}} \sin \theta_{\mathrm{i}}-n_{\mathrm{a}} \sin \theta_{\mathrm{r}}$ & $\theta_{\mathrm{r}}$ & $n_{\mathrm{g}} \sin \theta_{\mathrm{i}}-n_{\mathrm{a}} \sin \theta_{\mathrm{r}}$ & $\theta_{\mathrm{r}}$ & $n_{\mathrm{g}} \sin \theta_{\mathrm{i}}-n_{\mathrm{a}} \sin \theta_{\mathrm{r}}$ & $\theta_{\mathrm{r}}$ \\
\hline R-trained & $15^{\circ}$ & 0.36 & $25^{\circ}$ & 0.79 & $35^{\circ}$ & 0.28 & $34^{\circ}$ \\
T-trained & $3^{\circ}$ & -0.05 & $7^{\circ}$ & 0.14 & $20^{\circ}$ & -0.26 & $19^{\circ}$ \\
$p(t$ test $)$ & 0.02 & $<0.001$ & 0.02 & $<0.001$ & 0.06 & 0.005 & 0.27 \\
\hline \hline
\end{tabular}

"I liked the analogies. Made it very easy to understand without a lot of math!"

"The analogy really helped. Using the tires of the car to see which end left the medium first really helped me understand the direction it would exit."

"I love the examples of the wheels. I was not really sure how the light is refracted; I just memorized. But now I understand why the light is refracted that way."

"I did not get it until the professor used the car analogy."

In experiment 1,35\% of the participations spontaneously mentioned how helpful the car analogy was. In experiment 2 , exactly half of the students mentioned their appreciation for the car analogy. The fact that the analogy helped the students understand the principle of refraction is an interesting result itself. However, for us the most important aspect of these data is the fact that the students entered the testing phase feeling that they had a clear and deep understanding of how to implement the refraction principle.

\section{B. Experiment 1: Algebra students 1. General example bias}

In examining the results, the first question we asked is, Is there a general bias to students' responses that can be traced back to the training example they saw? Since R-trained participants saw the exiting ray bending upwards, while T-trained participants saw the exiting ray of the example bending downwards, we might expect a general upward bias to R-trained participants' responses, relative to those who were trained with the triangle example. This turns out to be the case, as shown in Table III.

We measured each student's angle of refraction at the first interface (air to glass in questions 1-3, glass to air in question 4). In questions 1-3 we also measured the angle of incidence to the second glass-air interface and the second refracted angle from glass to the air. We then developed a quantitative measure of their upward or downward bias at the second interface as follows: We defined our measure $m: m=n_{\mathrm{g}} \sin \theta_{\mathrm{i}}-n_{\mathrm{a}} \sin \theta_{\mathrm{r}}$, where we used the refractive index of glass, $n_{\mathrm{g}}=1.5$, and the refractive index of air, $n_{\mathrm{a}}=1$. We want to compare how much each participant bent their refracted ray down or up relative to the incident ray at the second interface. Using a measure comprised out of Snell's law is the simplest and most obvious way to measure how much the refracted ray is bent relative to the incident ray.

There are three interesting patterns that can be inferred from Table III. (a) In questions 1-3, there is a significant or marginally significant difference between the two groups' first refracted angle (air-glass). Although this could be interpreted as a manifestation of the specificity effect at the first interface, this difference is likely attributable

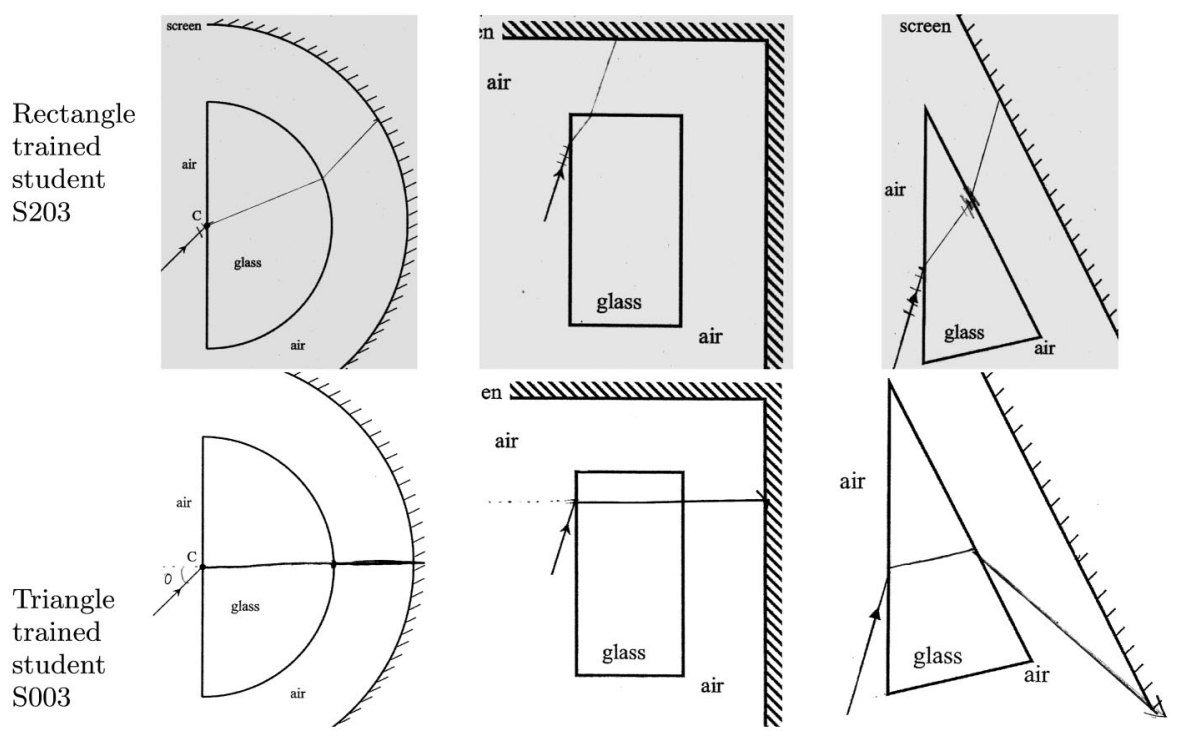

FIG. 3. Two subjects' responses to test questions 1,2, and 3 . 
TABLE IV. Evidence of specificity in algebra students.

\begin{tabular}{lccccccc}
\hline \hline & \multicolumn{2}{c}{ Question 1, $D$ prism } & \multicolumn{2}{c}{ Question 2, Rectangle prism } & \multicolumn{2}{c}{ Question 3, Triangle prism } \\
& R-trained & T-trained & R-trained & T-trained & R-trained & T-trained \\
\hline Correct & 3 & 5 & 3 & 5 & 8 & 6 \\
Specificity violation & 8 & 1 & 8 & 0 & 1 & 1.0 \\
$p$ (Fisher exact test) & & 0.05 & & & 0.03 & & 0 \\
\hline \hline
\end{tabular}

to T-trained participants' tendency to make the ray inside the prism horizontal (as in the training example), irrespective of context. Figure 3 shows a side-by-side comparison of the responses of two participants who learned through different examples. Student 003 's responses are typical of the group who saw the triangle example. Student 203's responses are typical of the group that saw the rectangle example. The overall bias at the first air-glass interface led us to modify the training example for the triangle group in experiment 2. (See Fig. 2.) (b) Another upward or downward bias can be seen at the second glass-air interface in questions 1-3. (c) R-trained and T-trained participants do not show any significant example bias in their responses to question 4.

\section{Specificity}

The general upward or downward bias of responses on questions 1-3 is interesting, but only suggestive of the specificity effect we expected. It simply may be the case that students, having seen only one training example, do not know any better than to make their responses match their training example as best they can. However, the results on question 4 do not support this hypothesis. If training with a rectangle or triangle prism led to a simple upward or downward bias in participants' responses, then we would expect to observe that bias in question 4. As noted in the previous section, there is no evidence of a bias in participants' responses to question 4.

More importantly, are there participants who demonstrate both evidence that they understand the principle of refraction and evidence that their knowledge is bound to the features of their training example? In this section we restrict our analysis to those participants who answered question 4 correctly (18 out of 31 , or $58 \%$ ). In our sample, 7 out of 15 (47\%) of the T-trained participants answered question 4 correctly and 11 out of $16(69 \%)$ of the R-trained participants answered question 4 correctly. These 18 participants were able to apply the principle of refraction at a single interface and therefore we assumed they understood the principle of refraction. In addition, all but one of these participants drew wave fronts and/or normal lines in questions 1,2 , and/or 3, suggesting that they were overtly attempting to apply the ideas they had just learned in a principled way. (Note the normal lines drawn by student 003 and wave fronts drawn by student 203 in Fig. 3 for example.)
Table IV shows a side-by-side comparison of R-trained and T-trained students' responses to questions 1-3. In each question, students' responses were coded "correct" if they bent all the light rays in the correct direction at each interface. If a student drew a pattern of rays that (a) matched their training example and (b) violated the refraction principle at one interface, we coded the response as a "specificity violation." Recall that all these students could reliably apply the refraction principle at a single interface (question 4).

These data show that R-trained students demonstrated significantly more specificity violations in questions 1 and 2. In question 3 , there was no significant difference between the groups. The responses shown in Fig. 3 are typical of responses in this group. It is interesting to note that in the triangle prism case, student 003 drew a pattern of light rays that matched the superficial features of her training example, but also did not violate the refraction principle just learned. It is therefore impossible to tell whether she was implementing principled knowledge or transferring surface features of her training example into the new situation, or both. Her response is typical of the T-trained group. A priori we had not expected that the horizontal ray inside the triangle prism used in training (Fig. 1) would be so salient and bias the T-trained groups' responses to such an extent.

Figure 3 shows examples of what we call the specificity effect. The first row shows the responses of student 203 (a R-trained participant) to questions 1, 2, and 3. Note how he draws a reasonable ray diagram for question 3 , but incorrectly reverses the direction of the exit ray in questions 1 and 2 to align his response with his training example (Fig. 1).

In contrast, consider the response of student 003 (a T-trained student) to questions 1,2 , and 3 shown in the second row of Fig. 3. While her responses to questions 1 and 2 are incorrect, since participants did not learn the numerical form of Snell's law, we consider that she is reliably instantiating the principle at both prism interfaces. She makes the light ray bend towards the normal at the first interface and remain undeviated at the second. From the overall responses of the T-trained participants, it appears that the horizontal segment of the ray diagram in the training example (Fig. 1) is extremely salient and appears to bias all their responses. For this reason, T-trained participants' responses to question 3 provide a less convincing case for the specificity effect since the ray drawn inside the prism struck the second interface slightly above the 
TABLE V. Summary of the angles and measures for calculus students.

\begin{tabular}{|c|c|c|c|c|c|c|c|}
\hline Question & \multicolumn{2}{|c|}{$\begin{array}{c}\text { Question 1, Rectangle } \\
\text { prism }\end{array}$} & \multicolumn{2}{|c|}{$\begin{array}{c}\text { Question 2, Triangle } \\
\text { prism }\end{array}$} & \multirow{3}{*}{$\begin{array}{c}\text { Question 3, Trap. } \\
\text { parallel part } \\
\text { Glass-Air } \\
\theta_{\mathrm{r}} \\
\end{array}$} & \multirow{3}{*}{$\begin{array}{c}\text { Question 4, Trap. } \\
\text { triangle part } \\
\text { Glass-Air } \\
\theta_{\mathrm{r}} \\
\end{array}$} & \multirow{3}{*}{$\begin{array}{c}\text { Question 5, Single } \\
\text { interface } \\
\text { Glass-Air } \\
\theta_{\mathrm{r}} \\
\end{array}$} \\
\hline Interface & Air-Glass & Glass-Air & Air-Glass & Glass-Air & & & \\
\hline Measure & $\theta_{\mathrm{r}}$ & $n_{\mathrm{g}} \sin \theta_{\mathrm{i}}-n_{\mathrm{a}} \sin \theta_{\mathrm{r}}$ & $\theta_{\mathrm{r}}$ & $n_{\mathrm{g}} \sin \theta_{\mathrm{i}}-n_{\mathrm{a}} \sin \theta_{\mathrm{r}}$ & & & \\
\hline R-trained & $28^{\circ}$ & 0.70 & $37^{\circ}$ & 0.25 & $25^{\circ}$ & $23^{\circ}$ & $55^{\circ}$ \\
\hline T-trained & $32^{\circ}$ & 0.33 & $37^{\circ}$ & 0.05 & $23^{\circ}$ & $52^{\circ}$ & $54^{\circ}$ \\
\hline$p(t$ test $)$ & 0.33 & 0.003 & 0.96 & 0.05 & 0.8 & 0.001 & 0.86 \\
\hline
\end{tabular}

normal. Even so, student 003 drew a sharply downwardangled exit ray.

\section{Experiment 2: Calculus students}

Because of the unexpected salience of the horizontal ray in the triangle prism example (Fig. 1) we modified the training example for the triangle-trained students so that the ray inside the prism was not horizontal (see Fig. 2). In all other respects, the training phase was the same as experiment 1 . We changed the test questions in order to better capture evidence for specificity. In particular, we did not provide any normal line in question 5, requiring students to apply the refraction principle entirely on their own (see Table II).

\section{General example bias}

As in experiment 1 , we measured the angle of incidence and refraction drawn by each student at each optical interface using a protractor. The results of these measurements are presented in Table V.

We can deduce the following results from Table V. (a) In every question, there is no evidence of example bias at the first interface, and no example bias between the R-trained and $\mathrm{T}$-trained students in question 5. This indicates that they all learned how to implement Snell's law qualitatively at a single interface. (b) There is evidence of an upward or downward bias at the second glass-air interface between the R-trained and T-trained students in questions 1, 2, and 4 , but no bias in question 3 .

\section{Specificity}

As before, we examined the subset of students who could answer question 5 correctly. Twenty-one R-trained students answered question 5 correctly; twenty-two T-trained students answered question 5 correctly. To evaluate whether or not there was evidence of the specificity effect, we restrict our analysis to questions 1 and 2 of the test (the rectangle prism and the triangle prism). Students who bent the light ray in the correct direction at both interfaces were coded as "correct" while students who drew a pattern of light rays that superficially matched their training example and, in doing so, violated the refraction principle by bending a light ray in the wrong direction were coded as "specificity violation."

We found a result that is both striking and puzzling. Out of the 21 R-trained students, 16 showed evidence of a specificity violation in question 1 (the rectangle prism), while all 21 drew correct light rays in question 2 (the

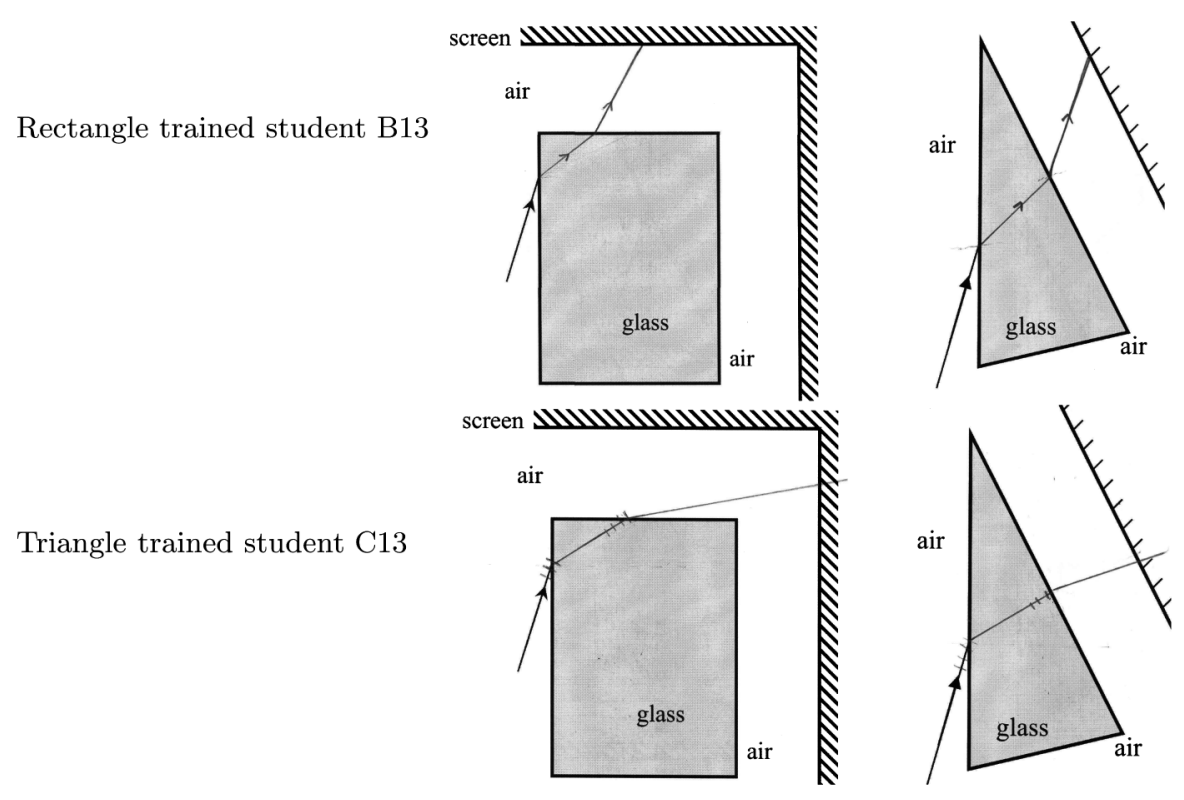

FIG. 4. Two participants' responses to test questions 1 and 2. 
triangle prism). This shows clear evidence of specificity. In contrast, out of the 22 T-trained students, 4 showed evidence of a specificity violation in question 2 (triangle prism) while 18 correctly answered question 1 (rectangle prism) correctly. Even more interestingly, we could identify an additional 4 T-trained students who showed evidence that they had drawn a specificity-biased light ray on question 2, but had erased it and drawn the correct light ray. In summary, we found specificity bias in both training conditions, yet the effect seemed to be much stronger in the R-trained group than in the T-trained group as evidenced by the larger number of students showing specificity violations.

Figure 4 shows examples of the specificity effect in 2 calculus physics students. The first row shows the responses of student B13 (a R-trained participant) to questions 1 and 2. Note how he draws a reasonable ray diagram for question 2, but incorrectly reverses the direction of the exit ray in question 1 to align his response with his training example (Fig. 2). C13 (a T-trained participant) also shows a specificity-biased response in question 2 , but correctly answers question 1 as shown in the second row of Fig. 4. Note how both participants seem to be trying to apply the principle they just learned by drawing normal lines and wave fronts at the interfaces.

\section{DISCUSSION AND IMPLICATIONS}

The results of experiments 1 and 2 show evidence that students who are able to implement the principle of refraction can be biased to give answers that violate that principle when the surface features of the test question superficially match the example used to illustrate the principle during training. Even when learning conceptually and interactively, participants tended to use surface features of their training example. When features of the test question were similar to the training example, this seemed to stimulate many participants to override or drop their principled knowledge and use surface features of the training example to answer that particular question. It also does not appear that this results is constrained to "weaker" algebra-based students. In fact, the calculus physics students' average American College Testing (ACT) math score was above the 95th percentile, indicating that these students are exceptional.

The implications of these results may extend beyond the example of refraction to many other areas of physics. If generalizable to other areas of introductory physics, the specificity effect has implications for how we measure what our students know and have learned. For example, if a multiple-choice distracter intentionally or inadvertently is made to look like an example students have encountered in their class, they may be biased to select that distracter, even though they displayed the ability to implement the principle in another assessment (for example, light bending at a single interface-like question 5). Likewise, when designing instruction, we need to think carefully about the examples we choose. First, if we use a single example to illustrate a principle, it is likely that students will learn the principle in too restrictive a context; thus, multiple examples would help students draw contrasts [11] on application of the principle in different contexts. Second, students may inadvertently focus on surface features of our examples that may appear extremely salient to them, but may serve no purpose in illustrating the physics principle we are trying to teach [12]. Further, this binding of examples and principles can be used to the instructor's advantage to help students learn about the principle. If learners think back and try to use an earlier example when it is not appropriate and are explicitly encouraged to consider why these similar cases are solved differently, they may learn much about the principle by comparing the two cases [13]

After the results of experiment 1, we hypothesized that the salience of the horizontal line was the reason why we did not see specificity in that experiment with the triangletrained group. However, experiment 2 did not fully support our idea. Experiment 2 shows a remarkable asymmetry in specificity. Sixteen rectangle-trained students showed specificity, while only four triangle-trained students (and possibly another four if we count the erasures) showed specificity. There are two possible reasons we can speculate about.

(1) Some aspect of the triangle example was simply "better" for student learning. It is possible that the triangle example did not have particular salient distracting surface features possessed by the rectangle example. Such questions are beyond the scope of this study and likely better suited to the domain of visual cognition in cognitive science.

(2) It is likely that many of the physics students, especially these exceptionally high-ability students in experiment 2 , had seen refraction before and seen the rectangle prism, so the triangle prism served as a contrasting case. During the instructional phase many of the triangle-trained students expressed an expectation that the light ray exiting the triangle prism should turn up rather than down as they tried to apply the car analogy in the context of the training example. Realizing that this violated the principle, they then drew a downward-angled ray. The fact that we saw evidence of T-trained students correcting their specificitybiased mistakes on question 2, and no evidence of this correction among the R-trained students, suggests that the T-trained students are thinking more carefully about what they are doing.

Finally, our results raise the question as to whether expert physicists are influenced by the specificity effect. How does a physicist recognize a physics problem as a particular type of problem (e.g., an energy conservation problem)? We speculate that experts use surface features of problems to help them cue their principled knowledge, but clearly surface features can fit various principles 
depending on context. Hence, it is the surface features in conjunction with context that create cuing priorities that facilitate experts' selection of specific principles. In other words, some principles are more likely applicable to a problem with certain surface features and specific context. If this is the case, it is possible that the expert can be distracted by surface features, just like the students in our study, especially when those surface features are used in unusual or uncommon contexts. Perhaps what makes an expert reliably choose principles to apply to problems is that, more often than not, certain surface features and contexts tend to go together, and by extensive experience experts become facile at recognizing those mappings so that appropriate principles are cued. When working in cutting edge problems, however, even experts can be surprised that their extensive knowledge may be insufficient to figure out what is going on, and these surprises often lead to significant discoveries [14]. Further research may examine whether there are other aspects to physics expertise that extend beyond the ability to cue principled knowledge in order to successfully solve a physics problem when principled knowledge is insufficient [15].

\section{ACKNOWLEDGMENTS}

We would like to thank Yuhfen Lin and Eugenia Etkina for their help. This material is based upon work supported in part by the Institute for Education Sciences of the U.S. Department of Education under Grant No. DE R305B070085. Any opinions, findings, and conclusions are those of the authors and do not necessarily reflect the views of the Institute for Education Sciences.
[1] B.H. Ross, Remindings and their effects on learning a cognitive skill, Cogn. Psychol. 16, 371 (1984).

[2] B. H. Ross, This is like that: The use of earlier problems and the separation of similarity effects, J. Exp. Psychol. Learn. Mem. Cogn. 13, 629 (1987).

[3] B. H. Ross, Distinguishing types of superficial similarities: Different effects on the access and use of earlier problems, J. Exp. Psychol. Learn. Mem. Cogn. 15, 456 (1989).

[4] M. Bassok and K. J. Holyoak, Interdomain transfer between isomorphic topics in algebra and physics, J. Exp. Psychol. Learn. Mem. Cogn. 15, 153 (1989).

[5] L. M. Reeves and R. W. Weisberg, The role of content and abstract information in analogical transfer, Psychol. Bull. 115, 381 (1994).

[6] J. P. Mestre, Learning and instruction in pre-college physical science, Phys. Today 44, No. 9, 56 (1991).

[7] A. Undreiu, D. Schuster, and B. Adams, in Proceedings of the 2008 Physics Education Research Conference, edited by C. Henderson, M. Sabella, and L. Hsu (American Institute of Physics, Melville, NY, 2008), pp. 211-214.

[8] J. Lobato, How design experiments can inform a rethinking of transfer and vice versa, Educ. Researcher 32, 17 (2003).

[9] N. S. Podolefsky and N.D. Finkelstein, Analogical scaffolding and the learning of abstract ideas in physics:
Empirical studies, Phys. Rev. ST Phys. Educ. Res. 3, 020104 (2007).

[10] A. Collins, J. S. Brown, and S. E. Newman, in Knowing, Learning, and Instruction. Essays in Honor of Robert Glaser, edited by L. B. Resnick (Lawrence Erlbaum Associates, Hillsdale, NJ, 1989), Chap. 14, pp. 453-494.

[11] F. Marton, Sameness and difference in transfer, J. Learn. Sci. 15, 499 (2006).

[12] A. F. Heckler, J.A. Kaminsky, and V.M. Sloutsky, in Proceedings of the $X X X$ Annual Conference of the Cognitive Science Society (Cognitive Science Society, Austin, TX, 2008), pp. 511-516.

[13] B. Rittle-Johnson and J. R. Star, Does comparing solution methods facilitate conceptual and procedural knowledge? An experimental study on learning to solve equations, J. Educ. Psychol. 99, 561 (2007).

[14] K. Dunbar, How scientists think in the real world: Implications for science education, J. Appl. Dev. Psychol. 21, 49 (2000).

[15] J.D. Bransford and D.L. Schwartz, in Review of Research in Education, edited by A. Iran-Nejad and P.D. Pearson (American Educational Research Association, Washington, DC, 1999), Vol. 24, Chap. 3, pp. 61-100. 